\title{
Modeling of nano-structured cathodes for improved lithium-sulfur batteries
}

\author{
Timo Danner ${ }^{\mathrm{a}, \mathrm{b}}$, Guanchen Zhu ${ }^{\mathrm{b}}$, Andreas F. Hofmann ${ }^{\mathrm{a}, \mathrm{b}}$, Arnulf Latz ${ }^{\mathrm{a}, \mathrm{b}, \mathrm{c}, *}$ \\ ${ }^{a}$ Institute of Engineering Thermodynamics, German Aerospace Center (DLR), \\ Pfaffenwaldring 38-40, 70569 Stuttgart, Germany \\ ${ }^{b}$ Helmholtz Institute Ulm for Electrochemical Energy Storage (HIU), 89069 Ulm, \\ Germany \\ ${ }^{c}$ Institute of Electrochemistry, University of Ulm, 89069 Ulm, Germany
}

\section{Abstract}

Some of the most remarkable improvements in the cycle life of Li-S batteries could be achieved by nano-structuring of the sulfur/carbon (S/C) cathode material. Meso- and micro-porous particles, carbon nano tubes, and other sophisticated structures are used to actively retain highly soluble polysulfides which are responsible for capacity fading and a short cycle life. In this article we present a detailed $1+1 \mathrm{D}$ continuum model of $\mathrm{S} / \mathrm{C}$ composite cathodes which is based on an ideal structure where all polysulfides are confined within meso- and micro-porous carbon particles. The model allows to correlate particle properties to cell performance and gives interesting insights for cell operation. Most interestingly we identified an additional overpotential caused by a transport of Li ions against a concentration gradient into the particle. Furthermore, the model can be used to find optimal structural and electrolyte properties such as pore volume, sulfur loading, solubility products,

\footnotetext{
${ }^{*}$ Corresponding author:

Email address: Arnulf.Latz@DLR.de ()

URL: http://www.dlr.de/tt/en/ ()
} 
and salt concentration.

Keywords: lithium-sulfur battery, modeling, meso/micro-porous carbon, sulfur encapsulation

\section{Introduction}

In recent years the demand for high energy density batteries was strongly driven by the developments on the market of portable electronic devices. At the moment mainly Li-ion batteries are used in cell phones, tablets, and laptop computers. However, the improvements in energy density in the last years were stagnating and a limit of the technology is intrinsically given by its intercalation chemistry. Next-generation Li batteries, such as lithiumsulfur (Li-S) and lithium-air, are based on the conversion of active materials. Therefore, higher energy densities can be attained which makes them also possible candidates for battery powered electric vehicles [1].

Li-S batteries are especially interesting because the electrodes are made of cheap, environmentally friendly, and sustainable materials. The battery typically consists of a porous sulfur/carbon (S/C) composite cathode, a porous separator soaked with a liquid organic electrolyte, and a lithium metal anode. Although research on Li-S batteries is now conducted for more than 50 years, several challenges obviated a successful commercialization of the technology. A serious problem which all Li metal based batteries have in common is the non-uniform Li stripping and plating upon cycling. In the long run this might lead to the formation of dendrites which can cause an internal short-circuit and pose a major security risk. The use of Si or Sn and their alloys as anode materials might help to improve this topic and is also 
suggested for Li-S batteries 24 4. Several issues are also associated with the sulfur cathode. $\mathrm{S}_{8}$ and the solid discharge product $\mathrm{Li}_{2} \mathrm{~S}$ have a poor electric conductivity. Moreover, the polysulfides which form during operation are highly soluble and are able to diffuse to the anode. This so-called 'shuttle effect' reduces the capacity and coulombic efficiency of the cells. In order to avoid these problems numerous approaches were suggested in the literature. They are summarized in recent review articles [5-10]. One approach is the optimization of the liquid electrolyte solution by tuning the solvent composition or the use of additives such as $\mathrm{LiNO}_{3}$ [11, 12]. Recently, it was also shown that an increase of salt concentration reduces the solubility of sulfides by the common ion effect and a promising cycling stability could be attained [13, 14]. Solid or polymer electrolytes were also suggested in order to block the polysulfide shuttle. However, this approach still suffers from the reduced ionic conductivity of the materials yet considerable improvements could be shown [8]. A lot of the recent work on Li-S batteries focuses on the active retention of polysulfides by a suitable nano-structuring of the electrode host materials, mostly carbon. This approach has the advantage that it additionally ensures a good electric contact of the solid sulfur. Mesoand micro-porous carbons [15-19], carbon nano tubes (CNTs) [20], graphene sheets [21], yolk/core-shell particles [22], and other structures were suggested to confine the polysulfides in the pore space of the cathode and remarkable improvements could be reported.

Taking into account the large body of experimental literature on Li-S batteries the number of modeling articles is relatively scarce. First continuum models of Li-S batteries were developed by Mikhaylik et al. [23] taking 
into account the shuttle effect by an empirical shuttle constant. Kumaresan et al. [24] present a detailed model describing the complex reduction mechanism of sulfur using a multi-species transport formulation of dissolved species. The same model was used by Ghaznavi et al. [25] 27] for intensive parameter studies which demonstrate the sensitivity of the model to parameter variations and the need for a reliable parametrization. A short-coming of the Kumaresan model is its missing ability to simulate the charge process. Fronczek et al. 28] proposed a similar model which allows for the simulation of battery charge and gives the opportunity to simulate electrochemical impedance spectra. This is an important feature since impedance simulations can be very helpful to clarify some of the aspects related to the reaction mechanism. Still, at the current state of research an unambiguous parametrization of the kinetic mechanism is difficult and Hofmann et al. [29] used a reduced set of equations for their mechanistic study of the polysulfide shuttle. It could be shown that the reduced kinetic model is able to represent the characteristic discharge behavior of Li-S batteries and the proposed shuttle mechanism gives a qualitative prediction of capacity degradation. A study taking into account the complex micro-structures as suggested in recent experimental articles could not be found in the modeling literature.

In this work we present a detailed continuum model of a S/C composite cathode consisting of meso- and micro-porous particles. First, a single-particle model is developed which employs a lumped kinetic mechanism as suggested by Hofman et al.. The particle model is coupled to a Li-ion battery model describing the macroscopic transport on cell level. An important assumption of our model is that dissolved polysulfides are confined within the meso- and 
microporous carbon particles. This corresponds to the ideal case which experimentalists are aiming for in their effort to find suitable nano-structures. The model is parametrized and validated based on experimental data from the literature [19]. It can be utilized to study different cell designs and transport phenomena which could not be observed in previous modeling work. Therefore, valuable insights on the operation and manufacturing of Li-S batteries with complex nano-structured cathodes can be obtained.

\section{Model development}

In this section the continuum models presented in this work are described in detail. The focus is set on the description of the Li-S particle model and its coupling to the macroscopic cell model. At the beginning we briefly discuss the kinetic mechanism of sulfur reduction presented in earlier work [24, 28, 29].

\subsection{Kinetic model}

The kinetic mechanism of sulfur reduction during discharge and oxidation upon charge is not yet totally understood. Several research groups try to shed some light on this issue using electrochemical [30 32] and analytical methods (UV-vis [33 35], XRD [36, 37], XANES [38, 39]) supported with information from quantum-chemical calculations [40, 41]. The proven reaction intermediates vary in the different studies which is also due to the different solvent systems employed in the measurements. Kumaresan et al. proposed a 5-step reduction from $\mathrm{S}_{8}^{(l)}$ down to $\mathrm{S}^{2-}$ where all intermediates except for $\mathrm{S}_{6}^{2-}$ are able to precipitate. The proposed mechanism is illustrated by black

arrows in Figure 1. The validity of this mechanism is under discussion and 
most likely other and/or additional reactions occur [32. Moreover, a large number of parameters need to be determined by a fit to experimental data. This makes an unambiguous determination of parameters very challenging. Ghaznavi et al. demonstrate in their intensive parameter studies [25]27] the strong sensitivity of simulation results on kinetic parameters.

In this qualitative study on transport effects in nano-structured electrodes we, therefore, use a reduced kinetic mechanism. Additionally, this approach minimizes the computational load for macroscopic battery cell simulations since the number of transport equations for intermediate species is smaller. Still, the characteristic shape of the discharge curve of a Li-S battery can be reproduced. This mechanism was already used in a previous publication of our group for the study of the poly-sulfide shuttle [29]. The proposed reduced reaction pathway is indicated by the green arrows in Figure 1. First, $\mathrm{S}_{8}^{(s)}$ dissolves in the liquid electrolyte

$$
\mathrm{S}_{8}^{(s)} \rightleftharpoons \mathrm{S}_{8}^{(l)} .
$$

$\mathrm{S}_{8}^{(l)}$ is then reduced via

$$
\begin{aligned}
1 / 4 \mathrm{~S}_{8}^{(l)}+e^{-} & \rightleftharpoons 1 / 2 \mathrm{~S}_{4}^{2-} \\
1 / 6 \mathrm{~S}_{4}^{2-}+e^{-} & \rightleftharpoons 4 / 6 \mathrm{~S}^{2-} .
\end{aligned}
$$

Finally, the solid discharge product $\mathrm{Li}_{2} \mathrm{~S}$ precipitates according to

$$
\mathrm{S}^{2-}+\mathrm{Li}^{+} \rightleftharpoons \mathrm{Li}_{2} \mathrm{~S}
$$

All reactions are assumed to be reversible and occur in the opposite direction upon charge. 
In our model we employ an expression for the reaction rate $r_{m}$ according to 42

$$
r_{m}=k_{f}^{0} \frac{a_{\text {ed. }}^{1-\alpha} a_{\text {prod. }}^{\alpha}}{\gamma_{\ddagger}}\left(e^{-\frac{\alpha}{R T} \Delta \bar{\mu}_{m}}-e^{\frac{1-\alpha}{R T} \Delta \bar{\mu}_{m}}\right),
$$

where $k_{f}$ is the reaction rate constant, $R$ is the ideal gas constant, and $T$ is temperature. $\alpha$ and $\gamma_{\ddagger}$ are the symmetry factor and the activity coefficient of the transition state, respectively. $\bar{\mu}_{i}$ is in our notation the electrochemical potential of species $i$. The terms $a_{\text {ed. }}$ and $a_{\text {prod. }}$ represent the products of the activities according to

$$
a_{\text {ed. }}=\prod_{\mathrm{i}=\text { ed. }} a_{i}^{\nu_{i, m}} \text { and } a_{\text {prod. }}=\prod_{\mathrm{i}=\text { prod. }} a_{i}^{\nu_{i, m}}
$$

where $\nu_{i, m}$ are the stoichiometric coefficients of the reaction. In this study all activity coefficients are assumed to be unity and activities follow as $a_{i}=$ $c_{i} / c_{\text {ref }}$. The reference concentration $c_{\text {ref }}$ is chosen to $1 \mathrm{~mol} / \mathrm{m}^{3}$ for all species and the activity of solids is per definition 1 . The electrochemical potential $\Delta \bar{\mu}_{m}$ of the chemical reaction $m$ follows as $\Delta \bar{\mu}_{m}=\sum_{i} \nu_{i, m} \bar{\mu}_{i}$. In the case of an electrochemical reaction $\Delta \bar{\mu}_{m}$ is given by

$$
\Delta \bar{\mu}_{m}=n F \eta=n F\left(\phi_{\text {elode }}-\phi_{\text {elyte }}-U_{\text {eq }}\right)
$$

where $n$ is the number of electrons transferred in the charge-transfer reaction $(\mathrm{CTR}), F$ is Faraday's constant, $\phi_{\text {elode }}$ the potential of the electrode, $\phi_{\text {elyte }}$ the potential in the electrolyte and $U_{\text {eq }}$ is the open circuit potential (OCP). It can be calculated according to

$$
U_{\text {eq }}=U_{\text {eq }}^{\ominus}+R T \ln \frac{a_{\text {ed. }}}{a_{\text {prod. }}},
$$


where $U_{\text {eq }}^{\ominus}$ is the OCP at reference conditions. For the chemical precipitation/dissolution reactions $\Delta \bar{\mu}_{m}$ can be written as

$$
\Delta \bar{\mu}_{m}=R T \ln \left(K_{\mathrm{sp}} \frac{a_{\text {prod. }}}{a_{\text {ed. }}}\right)
$$

where $K_{\mathrm{sp}}$ is the solubility product of dissolved species. A nucleation process of solid discharge products is taken into account by a modification of the specific surface area of the reaction $a_{m}^{v}$ (cf. Eq. (37] [24]). It is assumed that $a_{m}^{v}$ is proportional to the volume fraction of the precipitating solid. This phenomenological approach suppresses a formation of solid discharge products as long as their volume fraction is small and, thus, introduces a nucleation period. A more detailed treatment of precipitation kinetics using e.g. classical theory of nucleation and growth [43] could shed some more light on this interesting topic. However, this adds another level of complexity which is not feasible in this study.

\subsection{Particle model}

The magnification on the left-hand side of Figure 2 shows a schematic of the particle morphology studied in this article. It consists of a microporous carbon material in which the sulfur is initially uniformly distributed. The remaining pore space is totally impregnated with liquid electrolyte. The description of reaction and transport within the liquid phase of the microporous particle follows our earlier work on Li-S batteries [28, 29] and publications in the literature [24-27].

The flux $\dot{N}_{i}$ of species $i$ is described with dilute solution theory by

$$
\dot{N}_{i}=-D_{i}^{\mathrm{eff}} \frac{\partial c_{i}}{\partial r}-D_{i}^{\mathrm{eff}} c_{i} \frac{z_{i} F}{R T} \frac{\partial \phi_{\mathrm{elyte}}}{\partial r}
$$


where $c_{i}$ and $z_{i}$ are the concentration and charge number of species $i$. In dilute solution theory the interactions between dissolved species is neglected. This reduces the number of unknown parameters compared to the transport theory developed for concentrated solutions which is commonly used in the modeling of Li ion batteries (cf. Section 2.3 [44] [46] [47]). The effective diffusion coefficient $D_{i}^{\text {eff }}$ is modeled by the Bruggeman correlation

$$
D_{i}^{\mathrm{eff}}=D_{i}^{0} \varepsilon_{\text {elyte }}^{\beta_{p}}
$$

where $D_{i}^{0}$ is the bulk diffusion coefficient, $\varepsilon_{\text {elyte }}$ the volume fraction of the electrolyte and $\beta_{p}$ the Bruggeman coefficient in the micro-porous particle. A transport of species due to convection is neglected in this study. The conservation of mass of species $i$ in the radial coordinate $r$ follows as

$$
\frac{\partial \varepsilon_{\text {elyte }} c_{i}}{\partial t}=-\frac{1}{r^{2}} \frac{\partial}{\partial r}\left(r^{2} \dot{N}_{i}\right)+\sum_{m} a_{m}^{v} \nu_{i, m} r_{m},
$$

where $\sum_{m} a_{m}^{v} \nu_{i, m} r_{m}$ is a source term resulting from chemical and electrochemical reactions (cf. Eq. (5)). The potential in the electrolyte is calculated according to the conservation of charge under the assumption of electro-neutrality

$$
0=\sum_{i} z_{i} F\left(-\frac{1}{r^{2}} \frac{\partial}{\partial r}\left(r^{2} \dot{N}_{i}\right)+\sum_{m} a_{m}^{v} \nu_{i, m} r_{m}\right) .
$$

Since the conductivity of carbon is relatively high compared to the conductivity of the electrolyte solution the potential of the carbon particle is assumed to be constant.

The formation and dissolution of solid discharge products is described by

$$
\frac{\partial \varepsilon_{k}}{\partial t}=\frac{M W_{k}}{\rho_{k}} \sum_{m} a_{m}^{v} \nu_{k, m} r_{m}
$$


where $M W_{k}$ and $\rho_{k}$ are the molecular weight and density of phase $k$. The electrolyte is assumed to be ideally compressible and its volume fraction can be calculated according to

$$
\varepsilon_{\text {elyte }}=1-\sum_{k} \varepsilon_{k}
$$

Boundary conditions. At the particle center $(r=0)$ no-flux boundary conditions are applied for the transport of species $i$

$$
\left.\dot{N}_{i}\right|_{r=0}=0
$$

At the surface of the particle $\left(r=R_{\mathrm{p}}\right)$ it is assumed that only $\mathrm{Li}^{+}$ions are able to enter or leave the particle. The surface concentration and potential of the electrolyte are assumed to be constant

$$
\left.c_{\mathrm{Li}^{+}}\right|_{r=R_{\mathrm{p}}}=1250 \mathrm{~mol} / \mathrm{m}^{3} \text { and }\left.\quad \phi_{\text {elyte }}\right|_{r=R_{\mathrm{p}}}=0 \mathrm{~V} .
$$

All other species are forced to stay inside the particle and no-flux boundary conditions apply

$$
\left.\dot{N}_{i}\right|_{r=R_{\mathrm{p}}}=0
$$

This can be rationalized by an immobilization resulting either from the larger size of the molecules or a functionalization of the carbon surface [9]. In the cycling studies we incorporate a qualitative degradation effect by allowing the smallest polysulfide species $\mathrm{S}^{2-}$ to leave the particle. In a worst case scenario all $\mathrm{S}^{2-}$ ions are lost and the outside concentration is always close to zero

$$
\left.c_{\mathrm{S}^{2-}}\right|_{r=R_{\mathrm{p}}}=0 \mathrm{~mol} / \mathrm{m}^{3} .
$$


The electric current which is produced/consumed by the CTR inside the particle can be obtained by integration over the particle radius

$$
I=\frac{\int_{r=0}^{r=R_{\mathrm{p}}} 4 \pi r^{2} i_{F}(r) d r}{4 \pi R_{\mathrm{p}}^{2}},
$$

where $i_{F}(r)$ is the local Faradaic current produced by the CTRs and $I$ is the applied current density. The local Faradaic current $i_{F}(r)$ is given by

$$
i_{F}(r)=\sum_{\mathrm{CTRs}}-n F a_{m}^{v} r_{m}
$$

Eq. (20) gives the condition to determine the potential of the particle matrix $\phi_{\text {elode }}$.

\subsection{Macroscopic model}

For the description of the macroscopic battery cell we use a typical $1+1 \mathrm{D}$ continuum model based on porous electrode and concentrated solution theory [44, 45]. A schematic illustration of the modeling domain can be found in Figure 2.

The transport of the salt in the liquid electrolyte in direction of the macroscopic coordinate $x$ is given by

$$
\frac{\partial \varepsilon_{\mathrm{mac}} c_{e}}{\partial t}=\frac{\partial}{\partial x}\left(D_{e}^{\mathrm{eff}} \frac{\partial c_{e}}{\partial x}-\frac{i_{e}\left(1-t_{+}\right)}{F}\right),
$$

where $i_{e}$ is the ionic current in the electrolyte solution which can be described by a liquid-phase version of Ohm's law

$$
i_{e}=-\kappa_{\text {elyte }}^{\text {eff }} \frac{\partial \phi_{\text {elyte }}}{\partial x}-\kappa_{D}^{\text {eff }} \frac{\partial c_{e}}{\partial x} .
$$

$\kappa_{\text {elyte }}^{\text {eff }}=\kappa_{\text {elyte }} \varepsilon_{\text {mac }}^{1.5}$ is the effective ionic conductivity of the electrolyte and $\kappa_{D}^{\text {eff }}$ is an effective diffusional conductivity which follows as

$$
\kappa_{D}^{\mathrm{eff}}=\kappa_{\text {elyte }}^{\mathrm{eff}} \frac{2 R T\left(t_{+}-1\right)}{F c_{e}}\left(1+\frac{\partial \ln f_{e}}{\partial \ln c_{e}}\right) .
$$


Conservation of charge in the electrolyte is given by

$$
0=-\frac{\partial i_{e}}{\partial x}+a_{\mathrm{mac}}^{v} z_{\mathrm{Li}^{+}} F \dot{N}_{\mathrm{Li}^{+}}
$$

where $\dot{N}_{\mathrm{Li}^{+}}$is the flux of lithium ions at the electrode surface. In the cathode $a_{\mathrm{mac}}^{v}$ is determined by the particle size $R_{\mathrm{p}}$ and volume fraction $\varepsilon_{\mathrm{SC}}=1-\varepsilon_{\mathrm{mac}}$ of the sulfur-carbon composite

$$
a_{\mathrm{mac}}^{v}=\frac{3 \varepsilon_{\mathrm{SC}}}{R_{\mathrm{p}}} .
$$

Charge transport in the solid S/C composite can be described by Ohm's law

$$
0=-\kappa_{\text {elode }}^{\text {eff }} \frac{\partial \phi_{\text {elode }}}{\partial x}-a_{\mathrm{mac}}^{v} i_{F}^{\mathrm{mac}}
$$

where the effective conductivity of the electrode $\kappa_{\text {elode }}^{\text {eff }}=\kappa_{\mathrm{SC}} \varepsilon_{\mathrm{SC}}^{1.5}$ is again estimated by the Bruggeman correlation.

Boundary conditions. In the macroscopic cell model we assume no-flux boundary conditions of the electrolyte salt at $x=0$ (cathode current collector) which results in

$$
\left.\frac{\partial c_{e}}{\partial x}\right|_{x=0}=0 \quad \text { and }\left.\quad \frac{\partial \phi_{\text {elyte }}}{\partial x}\right|_{x=0}=0 .
$$

Due to charge conservation all current is transported in the solid S/C electrode and the boundary condition of Eq. 27 at $x=0$ is given by

$$
-\left.\kappa_{\mathrm{SC}} \frac{\partial \phi_{\text {elode }}}{\partial x}\right|_{x=0}=I,
$$

where $I$ is the current which is applied externally. At the interface between cathode and separator $\left(x=L_{\text {cat }}\right)$ all current is transported in the liquid 
electrolyte and the corresponding boundary condition in the $\mathrm{S} / \mathrm{C}$ composite is

$$
\left.\frac{\partial \phi_{\text {elode }}}{\partial x}\right|_{x=L_{\text {cat }}}=0
$$

The processes within the particle are coupled to the macroscopic model via the source terms of Eqs. (25) and (27). $\dot{N}_{\mathrm{Li}^{+}}$is described by

$$
\left.\dot{N}_{\mathrm{Li}^{+}}\right|_{r=R_{\mathrm{p}}}=-\left.D_{\mathrm{Li}^{+}}^{\mathrm{eff}} \frac{\partial c_{\mathrm{Li}^{+}}}{\partial r}\right|_{r=R_{\mathrm{p}}}-\left.D_{\mathrm{Li}^{+}}^{\mathrm{eff}} c_{\mathrm{Li}^{+}} \frac{z_{\mathrm{Li}^{+}} F}{R T} \frac{\partial \phi_{\mathrm{elyte}}}{\partial r}\right|_{r=R_{\mathrm{p}}} .
$$

In the derivation of the particle model it was explicitly assumed that the $\mathrm{Li}^{+}$ concentration and electrolyte potential on the particle surface are constant (cf. Eq. (17)). In the coupled model described in this paragraph the surface properties can be calculated with the help of the macroscopic cell model. The Faradaic current $i_{F}$ follows as in Eq. (20) by integration over the local reaction rates within the particle (see Eq. 210)

$$
\left.i_{F}^{\mathrm{mac}}\right|_{r=R_{\mathrm{p}}}=\frac{\int_{r=0}^{r=R_{\mathrm{p}}} 4 \pi r^{2} i_{F}(r) d r}{4 \pi R_{\mathrm{p}}^{2}} .
$$

At the anode we model the plating/stripping of lithium according to

$$
\mathrm{Li}^{+}+e^{-} \rightleftharpoons \mathrm{Li}^{(s)}
$$

The potential of the $\mathrm{Li}$ electrode is set as reference

$$
\left.\phi_{\text {elode }}\right|_{x=L_{\text {tot }}}=0 \mathrm{~V}
$$

and the corresponding flux of $\mathrm{Li}^{+}$ions is modeled by a reaction expression according to Eq. (5)

$$
r_{\mathrm{Li}}=k_{f}^{0} a_{\mathrm{Li}^{+}}^{1-\alpha}\left(e^{-\frac{\alpha F}{R T} \Delta \phi}-e^{\frac{(1-\alpha) F}{R T} \Delta \phi}\right) .
$$




\subsection{Parametrization and simulation methodology}

The parameters of the models described in the previous sections are obtained from the modeling articles on Li-S batteries and experimental data from the literature.

Structural parameters. Structural parameters are obtained from measurements on micro-porous sulfur-carbon composite materials [19]. The parameters of the single particle and full battery cell model are summarized in Table 1. Concentrations and volume fractions are initial values and change during the simulations. The dissolution and formation of phases has a strong influence on transport properties and active surface areas of the porous electrode. The effect on transport is implicitly taken into account by the Bruggeman correlation (cf. Eq. (11)). The Bruggeman coefficient of the macroscopic cell model is set to a standard value of 1.5. In the particle a high value of 10 is assumed in order to take into account the tortuous nanometer sized pores of the $\mathrm{S} / \mathrm{C}$ composite. In a separate study we investigated the influence of $\beta_{p}$ on battery operation, however, found only a negligible effect on simulation results.

A change of active surface area of the CTRs is described by an empirical correlation [24]

$$
a_{m}^{v}=a_{m, 0}^{v}\left(\frac{\varepsilon_{\text {elyte }}}{\varepsilon_{0}}\right)^{1.5} .
$$

The surface area of the dissolution/precipitation reactions is assumed to be

proportional to the volume fractions of the precipitating solid

$$
a_{\mathrm{S}_{8}^{(\mathrm{s})}}^{v}=a_{m, 0}^{v} \varepsilon_{\mathrm{S}_{8}^{(\mathrm{s})}} \quad \text { and } \quad a_{\mathrm{Li}_{2} \mathrm{~S}}^{v}=a_{m, 0}^{v} \varepsilon_{\mathrm{Li}_{2} \mathrm{~S}}
$$


thereby, empirically taking into account a nucleation period in the precipitation process of solid products [24].

Transport parameters. One of the most common liquid electrolyte systems for Li-S batteries is a mixture of DOL:DME. This solvent was also used in the experimental work which is the basis of our parameter fit [19]. Unfortunately, a consistent set of transport parameters could not be found in the literature. Therefore, transport parameters of the macroscopic model are taken from the literature for standard Li-ion battery electrolyte solutions [48, 49]. We are aware that carbonate based solvents are known to react with the polysulfide species in Li-S batteries [6]. However, recent studies on ultra-micro-porous carbon particles demonstrated a stable operation in carbonate based solvents for more than 100 cycles in case of a sufficient retention of polysulfides [18, 50].

In order to ensure a consistent parameterization of transport processes across the particle scale (dilute solution theory) and macroscopic scale (concentrated solution theory) we use in our calculations a constant value for the diffusion coefficient of $\mathrm{Li}^{+}$. It is calculated from the conductivity data of the electrolyte solution [48] based on dilute solution theory. Thus, we obtain similar conductivities in the macroscopic and particle model. The diffusion coefficients of the sulfur species are taken from Kumaresan et al. [24]. The pore size of the meso- and micro-porous S/C composite is less than $10 \mathrm{~nm}$. It was shown that transport of ions in micro-pores is strongly influenced by the electric double layer which has typically a thickness of $\approx 1 \mathrm{~nm}[51]$. Therefore, a deviation from bulk transport properties cannot be excluded if pore diameters are small. A detailed study of transport parameters in the 
micro-porous particle could give interesting insights on transport phenomena, however, it is out of the focus of this work. Transport parameters of this work are summarized in Table 2 .

Kinetic parameters. The kinetic and thermodynamic parameters of the reactions in the $\mathrm{S} / \mathrm{C}$ cathode were adjusted in order to reproduce the experimental data from the literature [19] (cf. Figure 3). As a reference for our fit we took the data of the $\mathrm{S} / \mathrm{C}$ composite electrode with a thickness of $100 \mu \mathrm{m}$. The measurements were recorded at a discharge rate of $\mathrm{C} / 10$ and a temperature of $298.15 \mathrm{~K}$. All reactions are parametrized with a symmetry factor $\alpha$ of 0.5. Simulation results obtained with the final set of parameters are discussed in detail in Section 3.1. A summary of kinetic and thermodynamic parameters can be found in Table 2.

Simulation methodology. The models are implemented in MATLAB and solved using ode15s. Computation time of the simulations is in the order of a few seconds up to minutes for the particle and coupled cell model, respectively.

\section{Results and discussion}

In this section we present results of our simulations with the single particle and macroscopic cell model. Parameter studies are performed to investigate the influence of particle micro-structure on battery performance. The models are also used for a qualitative study of capacity degradation which is presented at the end of this section. Possible strategies to improve the cycle life of Li-S batteries are also suggested and evaluated. 


\subsection{Model validation}

Kinetic and thermodynamic parameters of our model have been adjusted to meet the experimental data of meso- and micro-porous $\mathrm{S} / \mathrm{C}$ composite electrodes published in the literature (cf. Table 2 [19]). In order to reproduce the experimental geometry and conditions in our simulations the calculations are performed with the macroscopic cell model. Figure 3 shows simulated (lines) and experimental (symbols) charge and discharge curves for varying cathode thickness at room temperature. Although the parameters were only matched to discharge curves at a cathode thickness of $100 \mu \mathrm{m}$ we can report a good qualitative agreement of our simplified model with the experimental data at all simulated cathode thicknesses. Interestingly, we also observe in our simulations a comparatively smaller increase in capacity between a cathode thickness of 140 and $155 \mu \mathrm{m}$.

The simulated discharge curves exhibit the typical two-stage profile of Li-S batteries. Due to the lack of intermediate discharge products the simulation results have a sharp step between the first and second discharge plateau. The small dip in cell voltage at the beginning of the second plateau is caused by the nucleation of $\mathrm{Li}_{2} \mathrm{~S}$. The parameters of the precipitation reaction were chosen to reproduce the dynamics of this process as observed in the experimental data.

In general the predicted capacity of our simulations is slightly too high which can be explained by an incomplete sulfur utilization. This might be caused by the low electric conductivity of sulfur or an inhomogeneous distribution of the sulfur phase in the production process. This aspect is discussed in more detail in the following paragraph. Figure 3 also includes simulation results 
of battery charge. After a continuous increase in cell voltage we observe a plateau which can be linked to the precipitation of solid $\mathrm{S}_{8}$. Although the parameters were only tuned to represent battery discharge we can report a good agreement for battery charge as well. This result is assuring that our model allows for qualitative predictions of cell performance.

\subsection{Particle model}

In this section we present results obtained with the single-particle model. Figure 4 a) shows simulated discharge curves of particles with varying sulfur loading (uniform distribution). At low sulfur loading the discharge curves have a similar shape compared to the full cell model (cf. Figure 3) and a high sulfur utilization is predicted. Towards higher loadings a decrease in specific capacity can be observed. This can be assigned to a blocking of pores as indicated in the right panel of Figure 4.

Figure 5a) shows the mean polysulfide concentration as function of capacity. During discharge the concentration of dissolved polysulfides increases until $\mathrm{S}_{8}$ is consumed. At around the same time the precipitation of $\mathrm{Li}_{2} \mathrm{~S}$ kicks in and the polysulfide concentration is reduced. In order to guarantee electroneutrality within the particle Li ions have to migrate from the vicinity into the particle pore space. Therefore, the $\mathrm{Li}^{+}$concentration in the particle is proportional to polysulfide concentration. During discharge Li ions have to be driven into the particle against a gradient in concentration (cf. Figure 5 b)). This causes an additional transport overpotential as indicated by the potential difference given in the left panel of Figure 6. The $\mathrm{Li}^{+}$concentration difference increases with sulfur loading due to an increase in intermediate species concentration (Figure 5). As a consequence the resulting transport 
overpotential increases. It can be calculated from Eq. 10 to compensate the $\mathrm{Li}^{+}$gradient and is included as dotted line in Figure 6a). The solid line represents the overall potential difference in the liquid electrolyte between the particle surface and the first compartment inside the computational domain. This demonstrates that most of the potential difference is caused by the $\mathrm{Li}^{+}$ gradient. At the end of discharge the blocking of pores causes a steep increase in potential difference limiting the battery capacity. The overall magnitude of the overpotential is related to the concentration of intermediate sulfur dianions and, thus, depends on their solubility in the liquid electrolyte. Still, in order to guarantee electro-neutrality an influx of lithium is required and is so-to-say the price which has to be paid in order to encapsulate intermediate polysulfides. In the discharge curves the effect of the additional transport overpotential is not visible since the sulfur loading also affects the thermodynamics of the reactions.

A second aspect of the observed overpotential is that it is energetically favorable for polysulfides to leave the particle micro-structure. In fact the potential gradient induced by Li ions amplifies the drag on the polysulfide di-anions. This gives an explanation why already small cracks in hollow carbon spheres and similar structures are sufficient to trigger a rapid loss of polysulfides and, thus, capacity. Strategies to improve the cycle life based on observations on the particle level are given in Section 3.4 .

During the production process sulfur is impregnated into the carbon matrix at high temperatures. At these conditions sulfur has a low viscosity. It could be observed that sulfur preferentially impregnates the small micropores [9, 18]. This creates an inhomogeneous distribution of sulfur inside the 
particle matrix. In order to clarify the influence of this effect on discharge capacity we perform particle simulations with varying sulfur distribution but constant overall sulfur loading. In the case of our standard model where sulfur species are allowed to diffuse in the particle pore space no obvious effect of inhomogeneous sulfur distribution could be observed. In a second set of simulations we immobilized the sulfur species $\left(D_{\mathrm{S}_{x}}=0\right)$ at their position in the particle pore space. This can be rationalized by small pore sizes or chemisorption [9]. Results of the simulations are shown in Figure 6 b) together with the initial distribution (see inset). The simulations provide evidence that with increasing inhomogeneity sulfur is not totally reduced. This causes a decrease in discharge capacity. Our observation is in line with conclusions made in experimental studies [17, 16, 152] and serves as explanation for the deviations in discharge capacity obtained in our simulations on cell level (Section 3.1).

\subsection{Cell model}

The cell simulations allow to study the influence of microscopic particle properties on macroscopic battery performance. The left panel of Figure 7 shows discharge curves for various sulfur loadings. In addition to the simulations using the coupled particle model (solid lines) we include simulation results of a conventional composite electrode [29] (dashed lines) with corresponding macroscopic properties, such as electrode thickness, sulfur loading and overall porosity. Discharge curves of the meso-/micro-porous electrode show similar features as observed with the single particle model. In particular, we found a decrease in specific capacity at high sulfur loadings. This can be assigned to the blocking of transport pathways by the solid discharge 
product $\mathrm{Li}_{2} \mathrm{~S}$ on the particle scale. On the cell level the porosity is constant throughout the simulation. Figure $7 \mathrm{~b}$ ) shows the remaining average pore volume fraction inside the particle (solid lines) and composite electrode (dashed lines) for different sulfur loadings. It can be seen that the formation of discharge products generally proceeds faster close to the separator where the macroscopic lithium concentration is higher compared to the area close to the cathode current collector. In the case of the conventional composite electrode the available pore space is larger and, therefore, at the end of discharge only a small part of the pore space is occupied. This result shows that generally higher loadings can be achieved in meso- and macro-porous electrodes. However, on the price of a reduced cycle life due to degradation effects caused by the polysulfide shuttle.

In the literature an improved cycle life was reported for cells using a solvent/electrolyte system with high salt concentration [13, 14]. Simulations at varying salt concentration are presented in Figure 8. The discharge curves show a strong dependence on salt concentration and two major effects can be identified. First of all the cell voltage during the second plateau shows a maximum at a salt concentration of $3 \mathrm{M}$. This observation can linked to a strong decrease of the ionic conductivity at high salt concentrations due to a rise in viscosity. Similar observations could also be made in experimental studies [13]. The second effect is a decrease in battery capacity. Figure 8 b) shows the volume fractions of $\mathrm{S}_{8}$ (solid), $\mathrm{Li}_{2} \mathrm{~S}$ (dashed), and electrolyte (dashed dotted). It can be seen that the discharge stops if the porosity is around $40 \%$. In the case of a high salt concentration the dissolution of $\mathrm{S}_{8}$ and precipitation of $\mathrm{Li}_{2} \mathrm{~S}$ occurs inhomogeneous and preferentially at the 
cathode/separator interface. This is caused by a pronounced concentration gradient of the Li salt. As a result the capacity of the battery cell decreases. This observation is not fully in line with the experiments and more computational and experimental studies are needed to clarify the influence of particle structure in highly concentrated electrolyte solutions.

\subsection{Degradation and battery cycling}

A major challenge of Li-S batteries is the fast degradation of battery performance upon cycling. The most important reason is the loss of active material from the cathode to the anode due to the polysulfide shuttle. In this work we use the single particle model to study capacity degradation by allowing $\mathrm{S}^{2-}$ ions to leave the particle domain. Furthermore, we assume that all sulfur species outside the particle are immediately inactive. In the 'real' battery the sulfur is still trapped in the micro-structure of the cathode which delays the degradation of the battery. Therefore, this study can be regarded as a worst-case scenario. The left panel of Figure 9 displays the evolution of charge and discharge profiles of a single particle over 40 cycles. The graph illustrates a strong loss in capacity finally resulting in cell failure. The capacity of charge and discharge is displayed in Figure 9 b) in blue and red color, respectively. Initially the capacity decreases with a linear slope which becomes steeper at an increasing number of cycles. Since the battery is cycled at a constant current the loss in active material results into a relative increase in C-rate which is responsible for this effect.

The model allows for a qualitative prediction of the influence of system parameters on the lifetime of the battery. As pointed out earlier an important property of the electrolyte solution is the solubility product of polysulfides. 
The left graph of Figure 10 shows a variation of the solubility product of $\mathrm{Li}_{2} \mathrm{~S}$ over two orders of magnitude. In an electrolyte with a low solubility product $\mathrm{Li}_{2} \mathrm{~S}$ precipitates almost instantaneously. In this case most of the initial capacitance of the particle can be retained. However, if the solubility is high all of the capacity is lost after only very few cycles. This underlines the need for a development of suitable solvent/electrolyte systems. A similar result can be accomplished by increasing the salt concentration which affects polysulfide solubility by the common-ion effect [13, 14]. The influence of elevated salt concentrations on discharge performance has already been investigated in the previous paragraph. The focus of this section is set on the cycling stability of the system. Figure 10 b) shows a strong improvement of cycling stability towards higher concentrations. In this study we did not take into account other beneficial effects like an increased viscosity and ionic strength [13. or a reduced solubility due to interactions with the solvent molecules [40]. Still, the effect is quite remarkable. At this point it has to be reminded that a large salt concentration will lower the conductivity of the electrolyte solution which has a negative feedback on the performance of the battery. Moreover, the Li salt is rather expensive and a high concentration will also increase the price of the final battery.

Generally, our simulations show that it is probably not enough to use only a sophisticated micro-structure or only an improved electrolyte formulation to tackle the polysulfide shuttle. However, a combination of a suitable particle micro-structure with a well-balanced electrolyte system could help to solve some of the problems causing the fast decay of capacity. 


\section{Conclusions}

One of the largest challenges for modern Li-S batteries is their short cycle life. This is attributed to the high solubility of intermediate polysulfides and the resulting loss of active material due to migration of sulfur di-anions to the anode. Several approaches were suggested in the literature to tackle this problem where a nano-structuring of the cathode received the most attention.

In this article we present a continuum model of S/C cathodes consisting of meso- and micro-porous particles. The model is parametrized based on experimental data and the modeling literature on Li-S batteries. Our reduced kinetic model gives a qualitative agreement to the experimental data and allows for a prediction of battery performance and cycling stability. The simulations of a single-particle show that for high and non-uniform sulfur loadings the specific capacity effectively decreases. Most interestingly we identify an additional overpotential caused by the transport of $\mathrm{Li}^{+}$ions against a concentration gradient into the particle pore space. This also demonstrates the strong forces which will drive polysulfides out of the particle if they are not perfectly retained. We investigate this issue in degradation studies which show that the cycle life can be improved by using solvent systems with a low polysulfide solubility or a high salt concentration. However, simulations of the full battery cell show a detrimental effect of high salt concentrations on battery performance due to a lower ionic conductivity. Therefore, we conclude from our simulations that a well-balanced electrolyte system with a low solubility of polysulfides in combination with a sophisticated nanostructuring of the carbon host seems to be the most promising approach for an improved cycle life of Li-S batteries. 


\section{Acknowledgments}

The research leading to these results has received funding from the European Unions 7th Framework Program for research, technological development and demonstration (FP7/2007-2013) under grant agreement n³14282 (LISSEN). The authors thank the research partners at the Volkswagen Research Center Chiara Poggi and Stanislaw Ljahov for their support with micro-structural information and the scientific discussions. Timo Danner and Andreas Hofmann would especially like to thank Sören Thieme from the Fraunhofer IWS Dresden for sharing insights on his experimental data originating from the Project MaLiSu (EraNet).

[1] P. G. Bruce, S. A. Freunberger, L. J. Hardwick, J.-M. Tarascon, Li-O2 and Li-S batteries with high energy storage., Nat Mater 11 (1) (2012) 19-29. doi:10.1038/nmat3191.

[2] R. Elazari, G. Salitra, G. Gershinsky, A. Garsuch, A. Panchenko, D. Aurbach, Rechargeable lithiated silicon-sulfur (SLS) battery prototypes, Electrochem Commun 14 (1) (2012) 21-24. doi:10.1016/j.elecom. 2011.10.020.

[3] J. Hassoun, J. Kim, D. J. Lee, H. G. Jung, S. M. Lee, Y. K. Sun, B. Scrosati, A contribution to the progress of high energy batteries: A metal-free, lithium-ion, silicon-sulfur battery, J Power Sources 202 (2012) 308-313. doi:10.1016/j.jpowsour.2011.11.060.

[4] J. Hassoun, Y.-K. Sun, B. Scrosati, Rechargeable lithium sulfide electrode for a polymer tin/sulfur lithium-ion battery, J Power Sources 196 (1) (2011) 343-348. doi:10.1016/j.jpowsour.2010.06.093. 
[5] D. Bresser, S. Passerini, B. Scrosati, Recent progress and remaining challenges in sulfur-based lithium secondary batteries-a review., Chem Commun 49 (90) (2013) 10545-62. doi:10.1039/c3cc46131a.

[6] L. Chen, L. L. Shaw, Recent advances in lithium-sulfur batteries, J Power Sources 267 (2014) 770-783. doi:10.1016/j.jpowsour.2014. 05.111 .

[7] M. Barghamadi, A. Kapoor, C. Wen, A Review on Li-S Batteries as a High Efficiency Rechargeable Lithium Battery, J Electrochem Soc 160 (8) (2013) A1256-A1263. doi:10.1149/2.096308jes.

[8] M. Barghamadi, A. S. Best, A. I. Bhatt, A. F. Hollenkamp, M. Musameh, R. J. Rees, T. Rüther, Lithiumsulfur batteries the solution is in the electrolyte, but is the electrolyte a solution?, Energy Environ Sci 7 (12) (2014) 3902-3920. doi:10.1039/C4EE02192D.

[9] S. Evers, L. F. Nazar, New approaches for high energy density lithiumsulfur battery cathodes, Accounts Chem Res 46 (5) (2013) 1135-1143. doi:10.1021/ar3001348.

[10] S. S. Zhang, Liquid electrolyte lithium/sulfur battery: Fundamental chemistry, problems, and solutions, J Power Sources 231 (0) (2013) 153162. doi:10.1016/j.jpowsour.2012.12.102.

[11] Y. Mikhaylik, Electrolytes for lithium sulfur cells, US Patent 7,354,680 (Apr. 2008).

URL https://www.google.de/patents/US7354680 
[12] D. Aurbach, E. Pollak, R. Elazari, G. Salitra, C. S. Kelley, J. Affinito, On the Surface Chemical Aspects of Very High Energy Density, Rechargeable LiSulfur Batteries, J Electrochem Soc 156 (8) (2009) A694. doi: $10.1149 / 1.3148721$.

[13] E. S. Shin, K. Kim, S. H. Oh, W. I. Cho, Polysulfide dissolution control: the common ion effect., Chem Commun 49 (20) (2013) 2004-6. doi: $10.1039 / \mathrm{c} 2 \mathrm{cc} 36986 \mathrm{a}$.

[14] L. Suo, Y.-S. Hu, H. Li, M. Armand, L. Chen, A new class of Solvent-inSalt electrolyte for high-energy rechargeable metallic lithium batteries., Nat Commun 4 (2013) 1481. doi:10.1038/ncomms2513.

[15] X. Ji, K. T. Lee, L. F. Nazar, A highly ordered nanostructured carbonsulphur cathode for lithium-sulphur batteries., Nat Mater 8 (6) (2009) 500-6. doi:10.1038/nmat2460.

[16] X. Li, Y. Cao, W. Qi, L. V. Saraf, J. Xiao, Z. Nie, J. Mietek, J.-G. Zhang, B. Schwenzer, J. Liu, Optimization of mesoporous carbon structures for lithium-sulfur battery applications, J Mater Chem 21 (41) (2011) 16603. doi:10.1039/c1jm12979a.

[17] B. Zhang, X. Qin, G. R. Li, X. P. Gao, Enhancement of long stability of sulfur cathode by encapsulating sulfur into micropores of carbon spheres, Energy Environ Sci 3 (10) (2010) 1531. doi:10.1039/c002639e.

[18] S. Xin, L. Gu, N. H. Zhao, Y. X. Yin, L. J. Zhou, Y. G. Guo, L. J. Wan, Smaller sulfur molecules promise better lithium-sulfur batteries, J Am Chem Soc 134 (45) (2012) 18510-18513. doi:10.1021/ja308170k. 
[19] S. Thieme, J. Brückner, I. Bauer, M. Oschatz, L. Borchardt, H. Althues, S. Kaskel, High capacity micro-mesoporous carbonsulfur nanocomposite cathodes with enhanced cycling stability prepared by a solvent-free procedure, J Mater Chem A 1 (32) (2013) 9225. doi:10.1039/c3ta10641a.

[20] M. Rao, X. Song, H. Liao, E. J. Cairns, Carbon nanofiber-sulfur composite cathode materials with different binders for secondary Li/S cells, Electrochim Acta 65 (0) (2012) 228-233. doi:10.1016/j.electacta. 2012.01.051.

[21] H. Wang, Y. Yang, Y. Liang, J. T. Robinson, Y. Li, A. Jackson, Y. Cui, H. Dai, Graphene-wrapped sulfur particles as a rechargeable lithiumsulfur battery cathode material with high capacity and cycling stability, Nano Lett 11 (7) (2011) 2644-2647. doi:10.1021/nl200658a.

[22] Z. Wei Seh, W. Li, J. J. Cha, G. Zheng, Y. Yang, M. T. McDowell, P.-C. Hsu, Y. Cui, Sulphur-TiO2 yolk-shell nanoarchitecture with internal void space for long-cycle lithium-sulphur batteries., Nat Commun 4 (2013) 1331. doi:10.1038/ncomms2327.

[23] Y. V. Mikhaylik, J. R. Akridge, Polysulfide Shuttle Study in the Li/S Battery System, J Electrochem Soc 151 (11) (2004) A1969. doi:10. $1149 / 1.1806394$.

[24] K. Kumaresan, Y. Mikhaylik, R. E. White, A Mathematical Model for a Lithium-Sulfur Cell, J Electrochem Soc 155 (8) (2008) A576. doi: $10.1149 / 1.2937304$. 
[25] M. Ghaznavi, P. Chen, Sensitivity analysis of a mathematical model of lithium-sulfur cells: Part I: Applied discharge current and cathode conductivity, J Power Sources 257 (2014) 394-401. doi:10.1016/j. jpowsour.2013.10.135.

[26] M. Ghaznavi, P. Chen, Sensitivity analysis of a mathematical model of lithium-sulfur cells: Part II: Precipitation reaction kinetics and sulfur content, J Power Sources 257 (2014) 402-411. doi:10.1016/j. jpowsour.2013.12.145.

[27] M. Ghaznavi, P. Chen, Analysis of a Mathematical Model of LithiumSulfur Cells Part III: Electrochemical Reaction Kinetics, Transport Properties and Charging, Electrochim Acta 137 (2014) 575-585. doi: 10.1016/j.electacta.2014.06.033.

[28] D. N. Fronczek, W. G. Bessler, Insight into lithium-sulfur batteries: Elementary kinetic modeling and impedance simulation, J Power Sources 244 (2013) 183-188. doi:10.1016/j.jpowsour.2013.02.018.

[29] A. F. Hofmann, D. N. Fronczek, W. G. Bessler, Mechanistic modeling of polysulfide shuttle and capacity loss in lithium-sulfur batteries, J Power Sources 259 (2014) 300-310. doi:10.1016/j.jpowsour.2014.02.082.

[30] C. Barchasz, J.-C. Leprêtre, F. Alloin, S. Patoux, New insights into the limiting parameters of the Li/S rechargeable cell, J Power Sources 199 (0) (2012) 322-330. doi:10.1016/j.jpowsour.2011.07.021.

[31] N. A. Cañas, K. Hirose, B. Pascucci, N. Wagner, K. A. Friedrich, R. Hiesgen, Investigations of lithium-sulfur batteries using electro- 
chemical impedance spectroscopy, Electrochim Acta 97 (2013) 42-51. doi:10.1016/j.electacta.2013.02.101.

[32] Y.-C. Lu, Q. He, H. A. Gasteiger, Probing the Lithium-Sulfur Redox Reactions: A Rotating-Ring Disk Electrode Study, J Phys Chem C 118 (11) (2014) 5733-5741. doi:10.1021/jp500382s.

[33] M. U. M. Patel, R. Demir-Cakan, M. Morcrette, J. M. Tarascon, M. Gaberscek, R. Dominko, Li-S battery analyzed by UV/vis in operando mode, ChemSusChem 6 (7) (2013) 1177-1181. doi:10.1002/ CSSC.201300142,

[34] C. Barchasz, F. Molton, C. Duboc, Lithium/Sulfur Cell Discharge Mechanism: An Original Approach for Intermediate Species Identification, Anal Chem 84 (2012) 3973-3980. doi:10.1021/ac2032244.

[35] N. A. Cañas, D. N. Fronczek, N. Wagner, A. Latz, K. A. Friedrich, Experimental and Theoretical Analysis of Products and Reaction Intermediates of Lithium-Sulfur Batteries, J Phys Chem C 118 (23) (2014) 12106-12114. doi:10.1021/jp5013208.

[36] J. Nelson, S. Misra, Y. Yang, A. Jackson, Y. Liu, H. Wang, H. Dai, J. C. Andrews, Y. Cui, M. F. Toney, In Operando X-ray Diffraction and Transmission X-ray Microscopy of Lithium Sulfur Batteries, J Am Chem Soc 134 (14) (2012) 6337-6343. doi:10.1021/ja2121926.

[37] N. A. Cañas, S. Wolf, N. Wagner, K. A. Friedrich, In-situ X-ray diffraction studies of lithium-sulfur batteries, J Power Sources 226 (2013) 313319. doi:10.1016/j.jpowsour.2012.10.092. 
[38] M. Cuisinier, P. E. Cabelguen, S. Evers, G. He, M. Kolbeck, A. Garsuch, T. Bolin, M. Balasubramanian, L. F. Nazar, Sulfur speciation in Li-S batteries determined by operando X-ray absorption spectroscopy, J Phys Chem Lett 4 (19) (2013) 3227-3232. doi:10.1021/jz401763d.

[39] Y. Gorlin, A. Siebel, M. Piana, T. Huthwelker, H. Jha, G. Monsch, F. Kraus, H. A. Gasteiger, M. Tromp, Operando Characterization of Intermediates Produced in a Lithium-Sulfur Battery, J Electrochem Soc 162 (7) (2015) A1146-A1155. doi:10.1149/2.0081507jes.

[40] M. Vijayakumar, N. Govind, E. Walter, S. D. Burton, A. Shukla, A. Devaraj, J. Xiao, J. Liu, C. Wang, A. Karim, S. Thevuthasan, Molecular structure and stability of dissolved lithium polysulfide species., Phys Chem Chem Phys 16 (22) (2014) 10923-32. doi:10.1039/c4cp00889h.

[41] T. A. Pascal, C. D. Pemmaraju, D. Prendergast, X-ray spectroscopy as a probe for lithium polysulfide radicals, Phys Chem Chem Phys 17 (2015) 7743-7753. doi:10.1039/C4CP05316H.

[42] M. Z. Bazant, Theory of Chemical Kinetics and Charge Transfer based on Nonequilibrium Thermodynamics, Accounts Chem Res 46 (5). doi: $10.1021 / \operatorname{ar} 300145 \mathrm{c}$.

[43] B. Horstmann, T. Danner, W. G. Bessler, Precipitation in aqueous lithiumoxygen batteries: a model-based analysis, Energy Environ Sci 6 (4) (2013) 1299-1314. doi:10.1039/c3ee24299d.

[44] J. Newman, W. Tiedemann, Porous-electrode theory with battery applications, AIChE J 21 (1) (1975) 25-41. doi:10.1002/aic.690210103. 
[45] M. Doyle, T. Fuller, J. Newman, Modeling of galvanostatic charge and discharge of the lithium/polymer/insertion cell, J Electrochem Soc 140 (6) (1993) 1526-1533. doi:10.1149/1.2221597.

[46] A. Latz, J. Zausch, Thermodynamic consistent transport theory of Liion batteries, J Power Sources 196 (6) (2011) 3296-3302. doi:10.1016/ j.jpowsour.2010.11.088.

[47] A. Latz, J. Zausch, Multiscale modeling of lithium ion batteries: thermal aspects, Beilstein J Nanotechnol 6 (2015) 987-1007. doi:10.3762/ bjnano.6.102.

[48] L. O. Valoen, J. N. Reimers, Transport Properties of $\mathrm{LiPF}_{6}$-Based LiIon Battery Electrolytes, J Electrochem Soc 152 (5) (2005) A882. doi: 10.1149/1.1872737.

[49] A. Nyman, M. Behm, G. Lindbergh, Electrochemical characterisation and modelling of the mass transport phenomena in $\mathrm{LiPF}_{6}$-ECEMC electrolyte, Electrochim Acta 53 (22) (2008) 6356-6365. doi: 10.1016/j.electacta.2008.04.023.

[50] M. Helen, A. M. Reddy, T. Diemant, U. Golla-Schindler, J. R. Behm, U. Kaiser, M. Fichtner, Single step transformation of sulphur to $\mathrm{Li}_{2} \mathrm{~S}_{2} / \mathrm{Li}_{2} \mathrm{~S}$ in Li-S batteries, Sci. Rep. 5 (2015) 12146. doi:10.1038/ srep12146.

[51] M. Schmuck, M. Z. Bazant, Homogenization of the Poisson-NernstPlanck equations for ion transport in charged porous media, arXiv preprint.arXiv:1202.1916. 
[52] X. Tao, X. Chen, Y. Xia, H. Huang, Y. Gan, R. Wu, F. Chen, W. Zhang, Highly mesoporous carbon foams synthesized by a facile, cost-effective and template-free pechini method for advanced lithium-sulfur batteries, J Mater Chem A 1 (10) (2013) 3295. doi:10.1039/c2ta01213h.

[53] A. F. Holleman, E. Wiberg, Lehrbuch der anorganischen Chemie, 33rd Edition, Walter de Gruyter, Berlin, New York, 1995.

[54] D. R. Lide, CRC handbook of chemistry and physics, 87th Edition, CRC press, 2006. 


\begin{tabular}{|c|c|c|c|}
\hline & & Macroscopic & Particle \\
\hline \multirow{11}{*}{ Cathode } & Thickness/Radius & $100 \mu \mathrm{m}$ & $5 \mu \mathrm{m}$ \\
\hline & Volume elements & 10 & $10 / 5$ \\
\hline & Surface area & - & $1.348 \cdot 10^{9} \mathrm{~m}^{-1}$ \\
\hline & Bruggeman coefficient & 1.5 & 10 \\
\hline & Phases & & \\
\hline & Carbon & 0.6371 & 0.2174 \\
\hline & Binder & 0.1774 & - \\
\hline & $\mathrm{S}_{8}^{(\mathrm{s})}$ & - & 0.21 \\
\hline & $\mathrm{Li}_{2} \mathrm{~S}$ & - & $1 \cdot 10^{-4}$ \\
\hline & Electrolyte & 0.1855 & 0.5725 \\
\hline & $\left(\mathrm{Li}^{+} / \mathrm{A}^{-}, \mathrm{S}_{8}^{(1)}, \mathrm{S}_{4}^{2-}, \mathrm{S}^{2-}\right)$ & $(1250,19,0.0$ & $\left.6,1 \cdot 10^{-9} \mathrm{~mol} / \mathrm{m}^{3}\right)$ \\
\hline \multirow{7}{*}{ Separator } & Thickness & $30 \mu \mathrm{m}$ & - \\
\hline & Volume elements & 5 & - \\
\hline & Bruggeman coefficient & 1.5 & - \\
\hline & Phases & & \\
\hline & Polymer & 0.2 & - \\
\hline & Electrolyte & 0.8 & - \\
\hline & $\left(\mathrm{Li}^{+} / \mathrm{A}^{-}\right)$ & \multicolumn{2}{|c|}{$\left(1250 \mathrm{~mol} / \mathrm{m}^{3}\right)$} \\
\hline \multirow{5}{*}{ Anode } & Thickness & $1 \cdot 10^{-7} \mu \mathrm{m}$ & - \\
\hline & Volume elements & 1 & - \\
\hline & Surface area & $1 \cdot 10^{7} \mathrm{~m}^{-1}$ & - \\
\hline & Phases & & \\
\hline & Lithium & 1 & - \\
\hline
\end{tabular}

Table 1: Structural parameters of the standard geometry studied in this work. In the case of concentrations, volume fractions, and surface areas initial values are given which are subject to changes during the simulations. 


\begin{tabular}{llll}
\hline Parameter & Value & Meaning & Source \\
\hline
\end{tabular}

\section{Transport parameters}

\section{Electrolyte}

$\begin{array}{llll}\kappa_{\text {elyte }} & 1.161 \frac{\mathrm{S}}{\mathrm{m}}^{\dagger} & \text { Ionic conductivity } & {[48]} \\ t_{+} & 0.2498^{\dagger} & \text { Transference number } & {[49]} \\ \left(1+\frac{\partial \ln f_{e}}{\partial \ln c_{e}}\right) & 2.4296^{\dagger} & \text { Thermodynamic factor } & {[49]} \\ D_{\mathrm{Li}^{+}} & 1.237 \cdot 10^{-10} \frac{\mathrm{m}^{2}}{\mathrm{~s}} \dagger & \mathrm{Li}^{+} \text {diffusion coefficient } & {[48]} \\ D_{\mathrm{A}^{-}} & 1.237 \cdot 10^{-10} \frac{\mathrm{m}^{2}}{\mathrm{~s}} \dagger & \mathrm{A}^{-} \text {diffusion coefficient } & {[48]} \\ D_{\mathrm{S}_{8}} & 10 \cdot 10^{-10} \frac{\mathrm{m}^{2}}{\mathrm{~s}} & \mathrm{~S}_{8} \text { diffusion coefficient } & {[25]} \\ D_{\mathrm{S}_{4}^{2-}} & 1 \cdot 10^{-10} \frac{\mathrm{m}^{2}}{\mathrm{~s}} & \mathrm{~S}_{4}^{2-} \text { diffusion coefficient } & {[25]} \\ D_{\mathrm{S}^{2-}} & 1 \cdot 10^{-10} \frac{\mathrm{m}^{2}}{\mathrm{~s}} & \mathrm{~S}^{2-} \text { diffusion coefficient } & {[25]} \\ C^{-} & & & \end{array}$

\section{Carbon}

$\kappa_{\text {elode }} \quad 10 \frac{\mathrm{s}}{\mathrm{m}} \quad$ Electronic conductivity $\quad *$

\section{Kinetic parameters}

$\mathbf{S}_{8}^{(s)} \rightleftharpoons \mathbf{S}_{8}^{(l)}$

$\begin{array}{lclc}k_{f}^{0} & 1.484 \cdot 10^{-9} \frac{\mathrm{mol}}{\mathrm{m}^{2} s} & \text { Frequency factor of reaction } & * * \\ K_{\mathrm{sp}} & 19 & \text { Solubility product } & \text { [25] } \\ 1 / 4 \mathbf{S}_{8}^{(l)}+\mathbf{e}^{-} \rightleftharpoons 1 / 2 \mathbf{S}_{4}^{2-} & & \\ k_{f}^{0} & 2.044 \cdot 10^{-8} \frac{\mathrm{mol}}{\mathrm{m}^{2} s} & \text { Frequency factor of reaction } & * * \\ U_{\mathrm{eq}}^{\ominus} & 2.45 \mathrm{~V} & \text { OCP at reference conditions } & * * \\ 1 / 6 \mathbf{S}_{4}^{2-}+\mathbf{e}^{-} \rightleftharpoons 4 / 6 \mathbf{S}^{2-} & & \\ k_{f}^{0} & 2.044 \cdot 10^{-9} \frac{\mathrm{mol}}{\mathrm{m}^{2} s} & \text { Frequency factor of reaction } & * * \\ U_{\mathrm{eq}}^{\ominus} & 2.0 \mathrm{~V} & \text { OCP at reference conditions } & * * \\ \mathbf{S}^{2-}+\mathbf{L i}^{+} \rightleftharpoons \mathbf{L i}_{2} \mathbf{S} & & \end{array}$




\begin{tabular}{|c|c|c|c|}
\hline Parameter & Value & Meaning & Source \\
\hline$k_{f}^{0}$ & $7.419 \cdot 10^{-11} \frac{\mathrm{mol}}{\mathrm{m}^{2} s}$ & Frequency factor of reaction & $* *$ \\
\hline$K_{\mathrm{sp}}$ & $1 \cdot 10^{4}$ & Solubility product & $* *$ \\
\hline \multicolumn{4}{|c|}{$\mathbf{L i}^{+}+\mathbf{e}^{-} \rightleftharpoons \mathbf{L i} i^{(s)}$} \\
\hline$k_{f}^{0}$ & $4.084 \cdot 10^{-6} \frac{\mathrm{mol}}{\mathrm{m}^{2} \mathrm{~s}}$ & Frequency factor of reaction & 25 \\
\hline$U_{\text {eq }}^{\ominus}$ & $0.0 \mathrm{~V}$ & Li reference electrode & \\
\hline
\end{tabular}

\section{Thermodynamic parameters}

$\mathbf{S}_{8}^{(\mathbf{s})}$

$\begin{array}{llll}\rho_{\mathrm{S}_{8}^{(\mathrm{s})}} & 2070.4 \frac{\mathrm{kg}}{\mathrm{m}^{3}} & \text { Density } \\ M W_{\mathrm{S}_{8}^{(\mathrm{s})}} & 0.2565 \frac{\mathrm{kg}}{\mathrm{mol}} & \text { Molecular weight }\end{array}$

\section{$\mathrm{Li}_{2} \mathrm{~S}$}

\begin{tabular}{llll}
$\rho_{\mathrm{Li}_{2} \mathrm{~S}}$ & $1659.9 \frac{\mathrm{kg}}{\mathrm{m}^{3}}$ & Density & {$[54]$} \\
$M W_{\mathrm{Li}_{2} \mathrm{~S}}$ & $0.0459 \frac{\mathrm{kg}}{\mathrm{mol}}$ & Molecular weight & {$[54]$} \\
\hline
\end{tabular}

$\dagger$ value at $1250 \mathrm{~mol} / \mathrm{m}^{3}$ and $298.15 \mathrm{~K}$

* assumed parameter, ${ }^{* *}$ fit to experimental data

Table 2: Transport, kinetic, and thermodynamic parameters of species and phases considered in this work. 


\section{List of Figures}

1 Reaction mechanism at the cathode of a Li-S battery. Dis-

solved and solid polysulfide species are given in red and or-

ange color, respectively. Black arrows indicate the reaction

mechanism as proposed by Kumaresan et al.[24]. Our reduced

model [29] is represented by green arrows. . . . . . . . . . . . 40

$2 \quad$ Schematic illustration of the modeling domain. The direction

of the macroscopic modeling domain (cell level) is denoted by

$x$. The direction of transport in the particle model ('micro-

scopic') is given by $r$. . . . . . . . . . . . . . . . 41

$3 \quad$ Comparison between experiments (symbols) and simulation

results (lines) obtained with the cell model for varying cathode

thickness. . . . . . . . . . . . . . . . . . 41

$4 \quad$ Simulated discharge curves (left) and remaining pore volume (right) for a variation of sulfur loading. . . . . . . . . . . . . . 42

$5 \quad$ Left: Mean concentrations of polysulfides $\left(\mathrm{S}_{8}, \mathrm{~S}_{4}^{2-}, \mathrm{S}^{2-}\right)$ in the particle electrolyte volume obtained in simulations using the reduced kinetic model. Right: $\mathrm{Li}^{+}$concentration step between the particle surface and interior for various sulfur loadings. . . 42

6 Left: Potential step between particle surface and interior. Dashed lines give the transport overpotential which is needed to compensate the $\mathrm{Li}^{+}$concentration gradient. Right: Discharge curves of a single particle for a non-homogeneous sulfur distribution. Corresponding initial distributions are given in the inset. . . . . . . . . . . . . . . . . . 43 
$7 \quad$ Discharge curves (left) and electrolyte volume fractions (right) for a varying sulfur content. Solid lines represent simulation results obtained with our newly proposed particle model and dashed lines are results of a conventional composite model. . . 43

8 Influence of salt concentration on battery discharge. Left: Cell voltage as function of capacity. Right: Volume fractions of solid phases for selected concentrations. . . . . . . . . . . . . . 44

$9 \quad$ Simulated cycling behavior of a single S/C particle including a loss of $\mathrm{S}^{2-}$. Left: Discharge curves during cycling. Right: Capacity over number of cycles. . . . . . . . . . . . . . . . . . 44

10 Cycling simulations of the single particle model for varying $\mathrm{Li}_{2} \mathrm{~S}$ solubility (left) and initial salt concentration (right). The capacity degradation is caused by a loss of $\mathrm{S}^{2-}$ ions. . . . . . . 45 


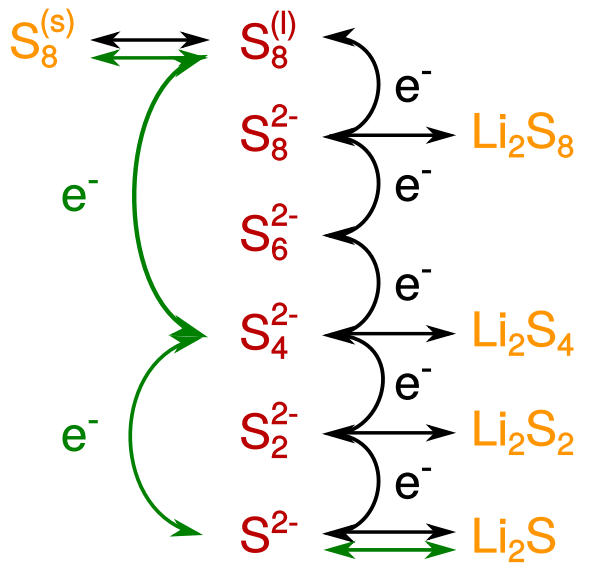

Figure 1: Reaction mechanism at the cathode of a Li-S battery. Dissolved and solid polysulfide species are given in red and orange color, respectively. Black arrows indicate the reaction mechanism as proposed by Kumaresan et al. 24]. Our reduced model [29] is represented by green arrows. 


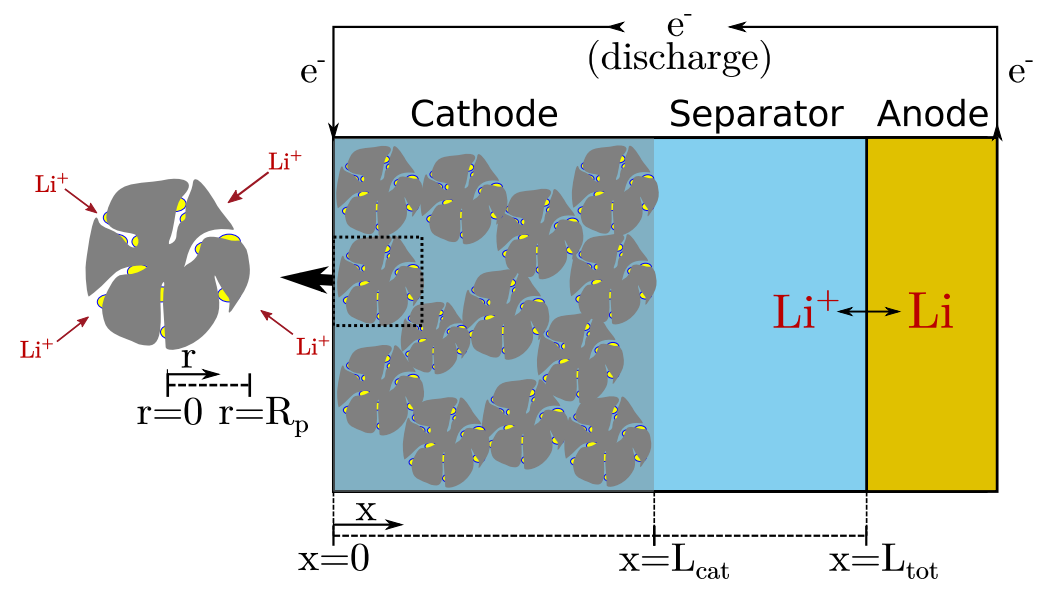

Figure 2: Schematic illustration of the modeling domain. The direction of the macroscopic modeling domain (cell level) is denoted by $x$. The direction of transport in the particle model ('microscopic') is given by $r$.

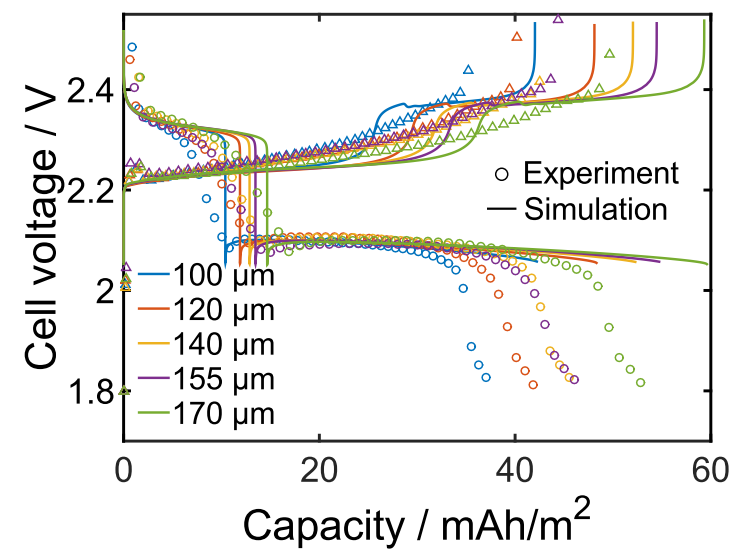

Figure 3: Comparison between experiments (symbols) and simulation results (lines) obtained with the cell model for varying cathode thickness. 

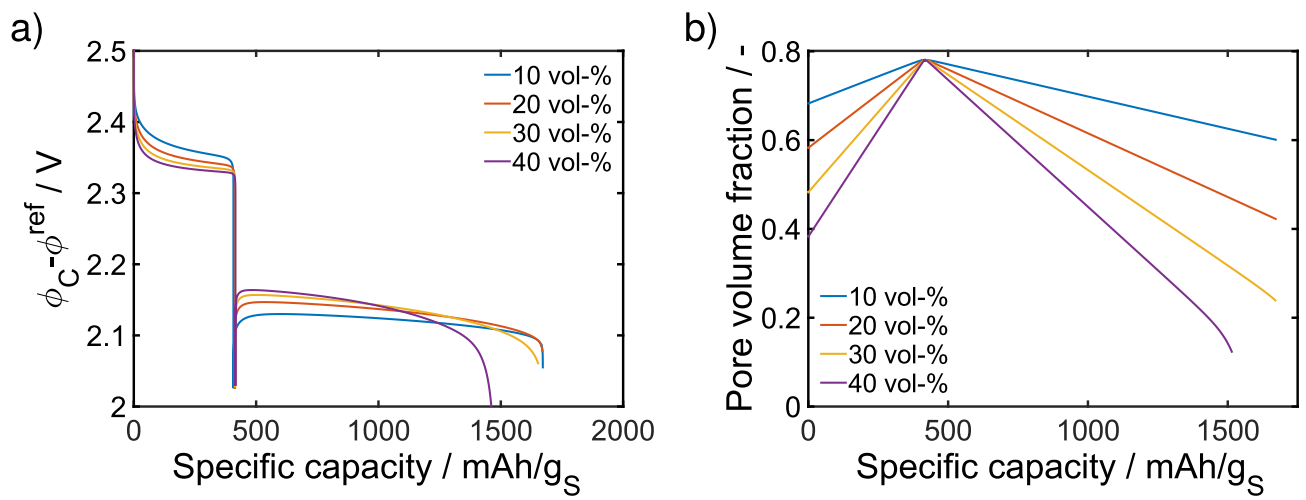

Figure 4: Simulated discharge curves (left) and remaining pore volume (right) for a variation of sulfur loading.
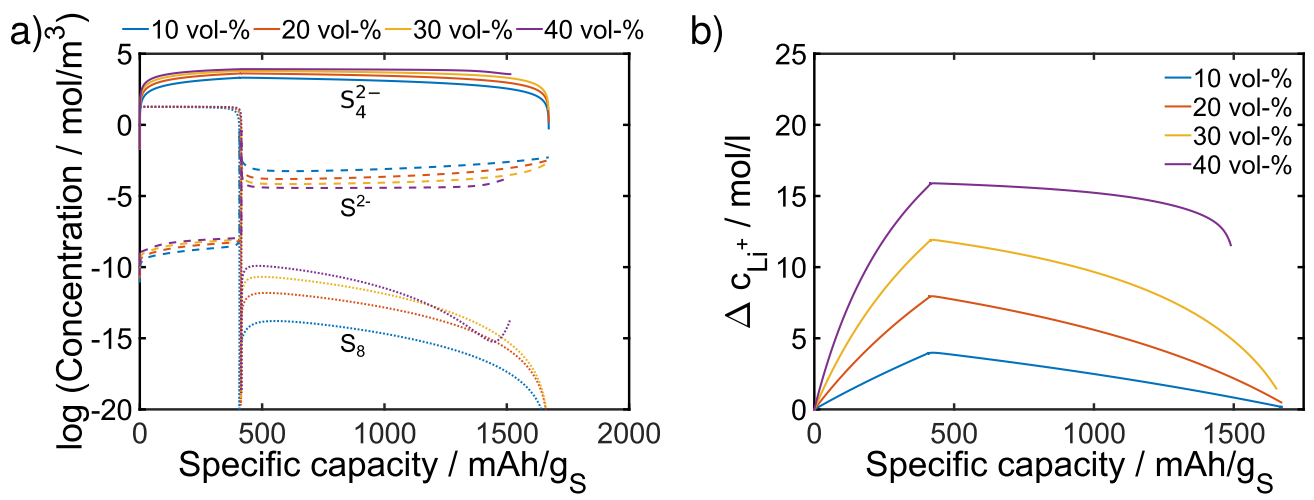

Figure 5: Left: Mean concentrations of polysulfides $\left(\mathrm{S}_{8}, \mathrm{~S}_{4}^{2-}, \mathrm{S}^{2-}\right)$ in the particle electrolyte volume obtained in simulations using the reduced kinetic model. Right: $\mathrm{Li}^{+}$concentration step between the particle surface and interior for various sulfur loadings. 
a)

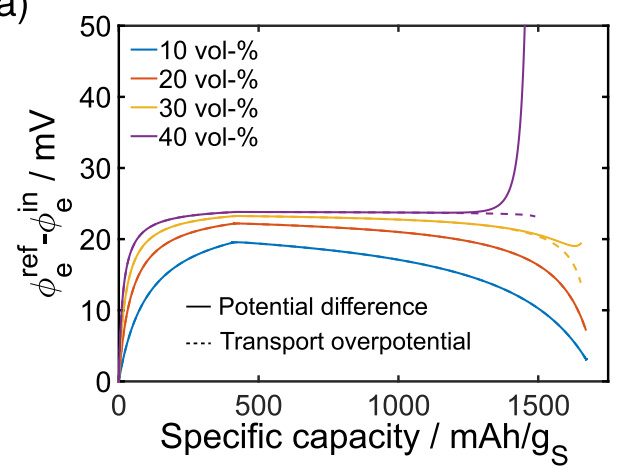

b)

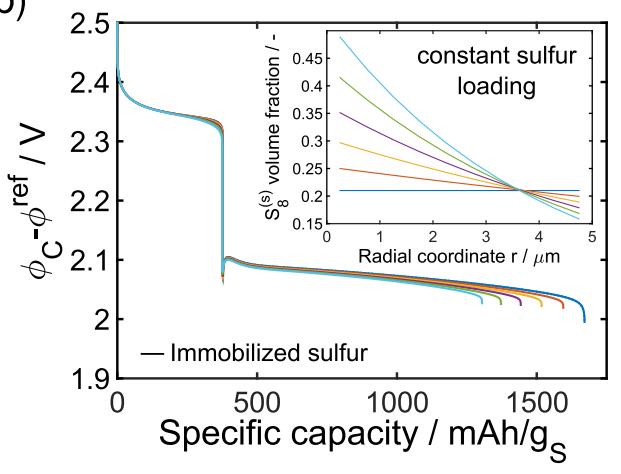

Figure 6: Left: Potential step between particle surface and interior. Dashed lines give the transport overpotential which is needed to compensate the $\mathrm{Li}^{+}$concentration gradient. Right: Discharge curves of a single particle for a non-homogeneous sulfur distribution. Corresponding initial distributions are given in the inset.

a)

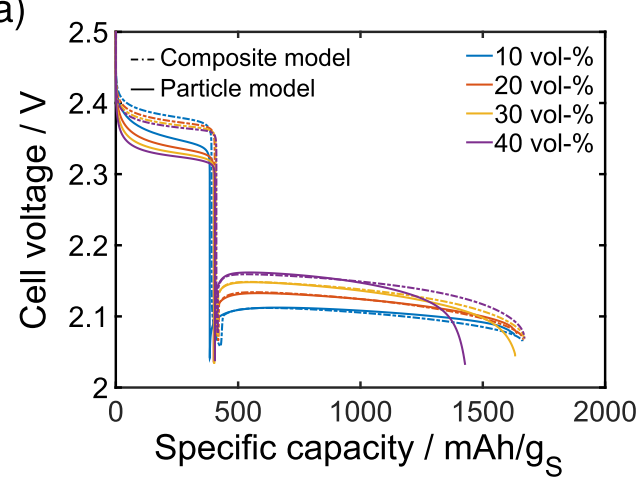

b)

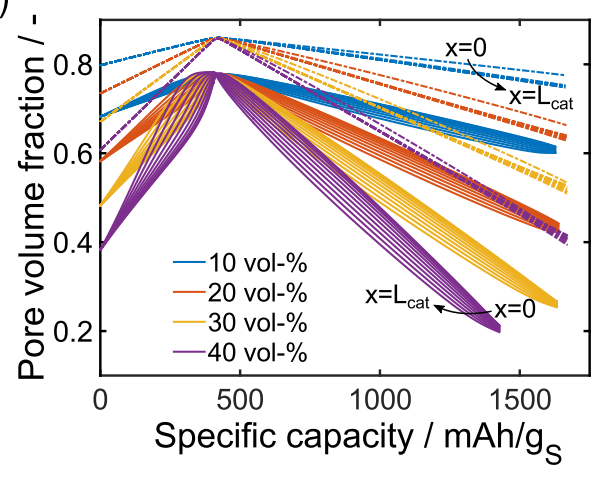

Figure 7: Discharge curves (left) and electrolyte volume fractions (right) for a varying sulfur content. Solid lines represent simulation results obtained with our newly proposed particle model and dashed lines are results of a conventional composite model. 

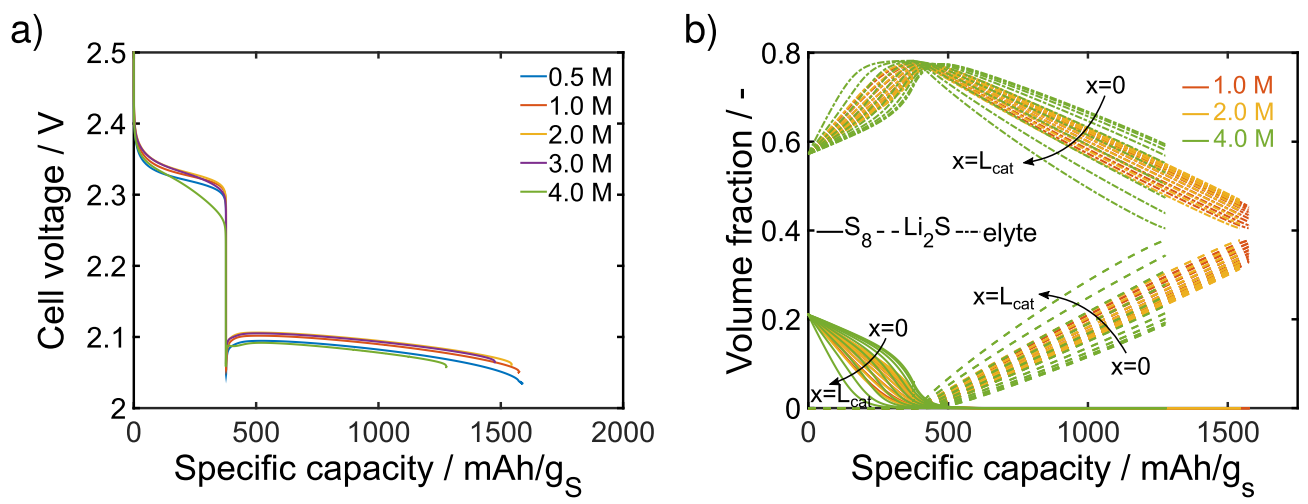

Figure 8: Influence of salt concentration on battery discharge. Left: Cell voltage as function of capacity. Right: Volume fractions of solid phases for selected concentrations.
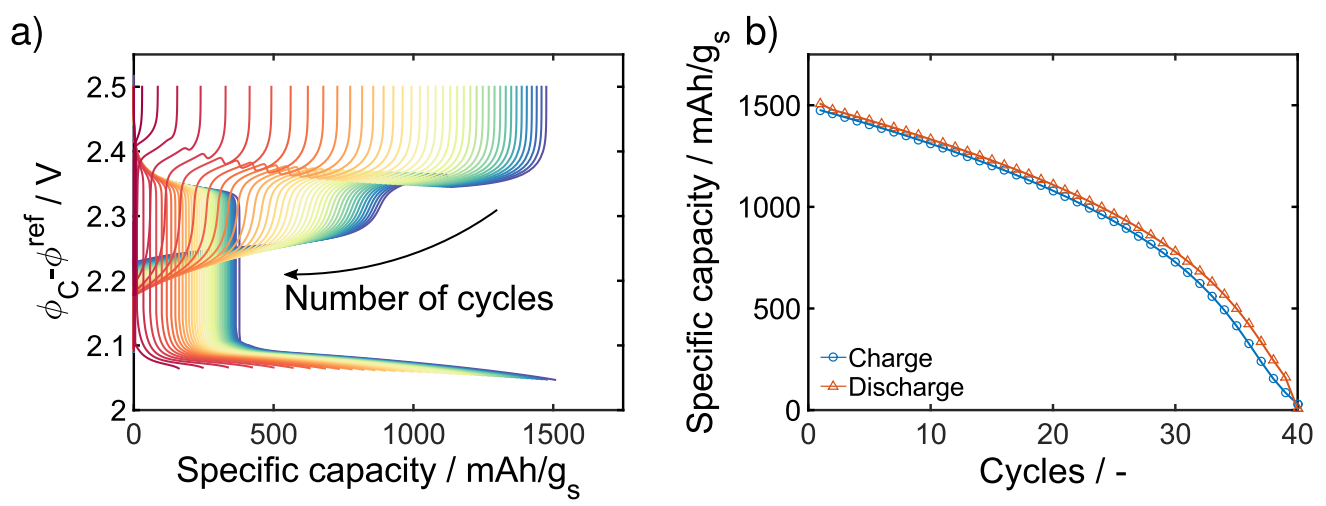

Figure 9: Simulated cycling behavior of a single S/C particle including a loss of $\mathrm{S}^{2-}$. Left: Discharge curves during cycling. Right: Capacity over number of cycles. 

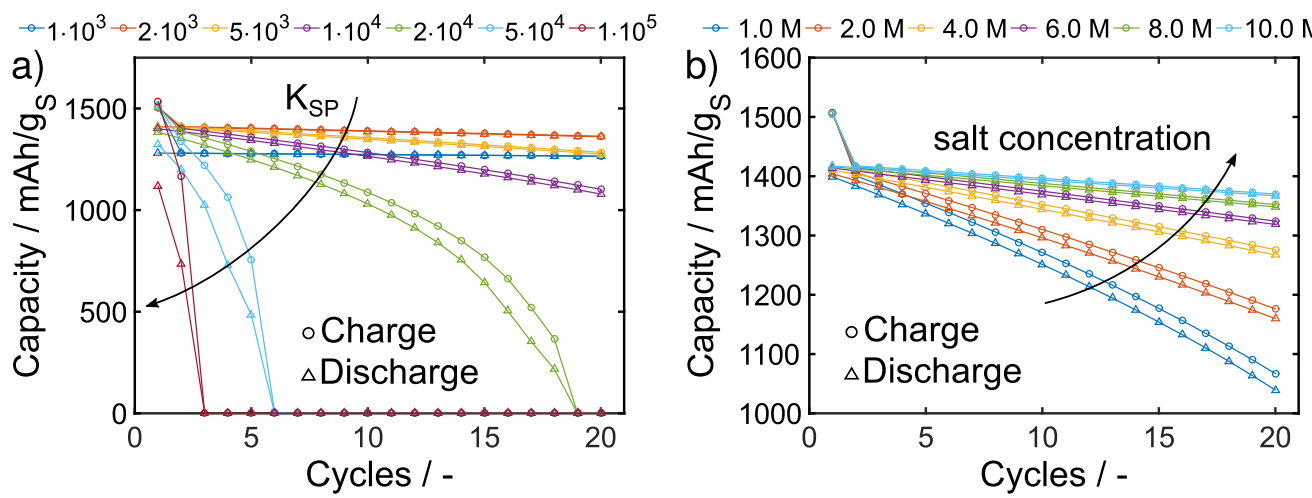

Figure 10: Cycling simulations of the single particle model for varying $\operatorname{Li}_{2} \mathrm{~S}$ solubility (left) and initial salt concentration (right). The capacity degradation is caused by a loss of $\mathrm{S}^{2-}$ ions. 